\title{
How psychiatrists can contribute to the public health
}

\author{
Robert Kendell
}

Public health has traditionally been concerned with the primary prevention of disease, either by eliminating its causes or by enhancing host resistance. Clean water supplies, efficient sewage and rubbish disposal, and refrigeration of food have largely eliminated the micro-organisms responsible for a wide range of infectious diseases, while anti-smoking campaigns have reduced mortality from cancer of the lung and coronary heart disease. Immunisation programmes have had a similarly dramatic effect on the incidence of many bacterial and viral diseases. Indeed, they have completely eliminated smallpox, and the polio virus seems likely to follow it into oblivion.

Sadly, most psychiatric disorders have not yet proved susceptible to this approach. Pellagra was quickly eliminated when epidemiological evidence established its dietary origins, the widespread use of penicillin soon eliminated general paralysis of the insane, and neonatal screening programmes now detect nearly all cases of phenylketonuria and congenital hypothyroidism in time to prevent their dire effects on intellectual development, but we still know too little about the causes of most psychiatric disorders for traditional public health measures to be feasible. That is why most public health physicians know so little psychiatry, although their recently acquired responsibilities for needs assessment and for advising purchasers are starting to alter this.

Although there is no prospect yet of introducing public health measures that would greatly reduce the incidence of schizophrenia, bipolar disorder, Alzheimer's disease or common neurotic disorders, there are a wide range of measures that either already have reduced, or could confidently be expected to reduce, the incidence of these and other psychiatric disorders to a worthwhile extent. The problem from a consultant psychiatrist's point of view is that most of the measures that are known to be effective are not under his or her control, and most of the psychological or social measures that he or she might take, or at least influence, are of uncertain value.

Amniocentesis, particularly if combined with routine biochemical screening of all pregnant women, has the potential to reduce the incidence of Down's syndrome. We can also safely assume that the widespread availability in the past 25 years both of effective contraceptive measures and of termination of pregnancy has prevented many unwanted children being born, and many children being born to mothers and families incapable of giving them adequate security, stability and affection, and that as a result many lifelong personality disorders have been prevented. Indeed, some cases of schizophrenia may have been prevented (Myhrman et al, 1996). Similarly, we can assume that a wide range of safety measures and legislation intended to minimise the risk of serious or fatal accidents have effectively reduced the incidence of post-traumatic stress disorder, even though those responsible for these safety measures had probably never even heard of PTSD. It is also possible that the incidence of schizophrenia in industrialised countries has been reduced over the past 30 years by improvements in obstetric care, although it is important to appreciate that it is still uncertain whether the incidence of schizophrenia has really declined, and not entirely clear that the raised incidence of obstetric complications associated with schizophrenia is causal rather than consequential. In all these situations, however, the measures involved

Robert Kendell was Professor of Psychiatry at the University of Edinburgh from 1974 to 1991 . For the next five years he was Chief Medical Officer in the Scottish Office and responsible for public health in Scotland. Currently, Dr Kendell is President of the Royal College of Psychiatrists, 17 Belgrave Square, London SW1X 8PG. 
are not within the ambit of psychiatry. They are the province of safety engineers, legislators, obstetricians and paediatricians.

\section{Levels of prevention}

Public health has traditionally distinguished three levels of prevention (Box 1). Primary prevention involves reducing the incidence of a disorder (the rate at which new cases occur). It is directed at people who are well but are at risk of developing the disorder and achieved either by eliminating the cause (e.g. asbestos or smoking) or by enhancing host resistance (e.g. childhood immunisation). Secondary prevention involves detecting the disorder at an early stage when treatment is likely to be more effective (e.g. screening for phenylketonuria or for cancer of the cervix). Tertiary prevention involves reducing the disability resulting from a disorder. It includes relapse prevention, and overlaps with both treatment and rehabilitation. The long-term treatment of schizophrenia with neuroleptics, of bipolar disorder with lithium or carbamazepine, and of recurrent depression with tricyclic antidepressants or lithium are, therefore, all examples of tertiary prevention. So too is family therapy to reduce high levels of 'expressed emotion' in the relatives of schizophrenics.

The distinction between high-risk and populationbased strategies is another fundamental public health concept. Population-based primary and secondary prevention strategies, like childhood immunisation programmes, are intended to embrace the whole population (even though some individuals may be at greater risk). High-risk strategies, like restricting screening for hypercholesterolaemia to middle-aged men who smoke or have a family history of heart disease, are focused on subgroups known to be at comparatively high risk.

\section{'Positive mental health'}

Partly because many psychiatric disorders are chronic conditions which can be ameliorated but rarely cured, there have been recurrent waves of enthusiasm for the adoption of measures which would, it was hoped, prevent mental disorders from ever developing by eliminating or reducing their presumed social and psychological causes. The Mental Hygiene Movement of the 1920s and 1930s and the Community Mental Health programmes of the Kennedy era in the USA were both intended as primary prevention programmes. Both
Box 1. Three levels of prevention

Primary prevention - reduces incidence (new cases)

Secondary prevention - detection at an early stage when treatment is more effective

Tertiary prevention - measures which reduce resulting disability

failed, at least in their major objectives, because their therapeutic assumptions had never been empirically validated beforehand.

The promotion of 'positive mental health' is the contemporary equivalent of these earlier enthusiasms. Its proponents seek to combat poverty, loneliness, low self-esteem, fear and hostility, and generally to promote tolerance, happiness and contemporary liberal middle-class values. Their sincerity and good intentions are not in doubt, but many of the measures they advocate are impracticable and most have never been shown to prevent any form of psychiatric disorder, still less to have the widespread benefits that their advocates assume. Despite its fine resonances it is also unclear what the slogan 'positive mental health' really means. It is intended to denote something much more important and worthy than simply the absence of illness and seems to have much in common with happiness and self-fulfilment. It also, presumably, implies the existence of a contrasting state of 'negative mental health', although this is never mentioned.

At least to the present author, health and sickness, like good and evil or light and darkness, constitute a single bipolar concept. If there were no such thing as disease or sickness, we would have no concept of health, and health can have no other meaning than the absence of sickness. To describe to what extent or in what way someone is sick, or liable to become so, automatically defines the extent to which they are healthy, and vice versa. I would also argue that in all fields of medicine, and above all where psychiatric disorders are concerned, we have plenty to do striving to eliminate overt illness without setting ourselves a target of achieving some indefinable, more distant goal of 'positive health'.

\section{Learning disability}

So far, this has been the most fruitful field for both primary and secondary prevention in psychiatry 
(Box 2), mainly because a large number of specific syndromes and causal agents have been identified. Improvements in obstetric care have greatly reduced the incidence of learning disability resulting from peripartum brain damage, although this has been offset to some extent by the increased survival chances of premature or low birth-weight infants. Literally hundreds of genetic disorders associated with learning disability have now been identified, but most are very rare, and genetic counselling is generally only feasible where there is an affected sibling or a family history of the condition. Vaccination of schoolgirls against rubella has greatly reduced the incidence of the congenital rubella syndrome, and avoidance of heavy drinking in pregnancy would prevent the fetal alcohol syndrome. Screening for phenylketonuria, a relatively common autosomal recessive disorder, now identifies almost all cases at birth, and a low phenylalanine diet throughout childhood largely prevents the associated cerebral damage.

At present, Down's syndrome and fragile $X$ syndrome present the most important preventive opportunities because of their comparatively high incidence. Amniocentesis, followed by termination of pregnancy if the fetus is found to be affected, has long been offered to all pregnant women over the age of 35 (a classical high-risk strategy) and within the next year or two the UK Department of Health may well decide to offer all women biochemical screening (the 'triple test' for alpha fetoprotein, chorionic gonadotrophin and unconjugated oestriol) in early pregnancy (Wald et al, 1992). As most Down's infants are born to mothers under the age of 35 this offers a prospect, for the first time, of effecting a substantial reduction in the number of children born with Down's syndrome. Fragile $X$ is responsible for $10 \%$ of learning-disabled males, and the recent identification of the gene opens the way to the detection of carrier females, genetic counselling and prenatal diagnosis of affected males.

Box 2. Learning disability is a fruitful field for both primary and secondary prevention

Prevention of birth injury by good obstetric care

Vaccination of schoolgirls against rubella

Neonatal screening for phenylketonuria and thyroid deficiency

Biochemical screening and amniocentesis for Down's syndrome

Screening carrier females for fragile $X$
Alzheimer's disease and other dementias

Despite the identification of several predisposing genes, primary prevention is not yet feasible for Alzheimer's disease. Although there is some intriguing evidence that aluminium is involved in the aetiology of the condition, there is no evidence that avoiding aluminium cooking utensils and drinking water with a high aluminium content reduces the incidence of the disease. Nor do people who consumed large quantities of aluminium hydroxide for years on end to alleviate dyspepsia appear to be at increased risk. There is, however, some evidence that intellectual activity maintained into old age provides an element of protection. Boxers' encephalopathy can, of course, be avoided by not boxing, and the incidence of multi-infarct dementia is reduced by the measures which reduce cerebrovascular disease in general, namely the detection and treatment of hypertension, stopping smoking and a low-fat diet. The identification of the gene for Huntington's chorea on chromosome 4 makes it possible to provide accurate genetic counselling for members of affected families and the eventual elimination of the disorder by prenatal diagnosis and termination of affected pregnancies, but the ethical and practical difficulties involved are profound.

\section{Schizophrenia}

Although there is indisputable evidence of a major genetic element in the aetiology of schizophrenia, no 'schizophrenia gene' has yet been identified. It also seems increasingly likely that several different genes with additive effects underlie the syndrome, which suggests that genetic counselling and primary prevention will not be easy, even after the responsible genes have been located. The fact that the concordance rate in monozygous twins is less than $50 \%$ is strong evidence that environmental factors are involved, and many lines of evidence suggest that these act mainly in utero or at the time of birth. The evidence is not yet strong enough, however, to form a basis for primary prevention strategies.

It is well established that schizophrenics have a higher incidence of obstetric complications of various kinds at the time of their birth than matched controls (Geddes \& Lawrie, 1995) but it is conceivable that these are secondary to the 
cerebral abnormalities underlying the syndrome rather than causal. It is equally well established that a long interval between the onset of the disorder in adolescence or early adult life and admission to hospital for treatment is associated with a poor long-term prognosis. This is generally assumed to be because an insidious onset and the absence of obvious precipitants are themselves linked with a poor prognosis, but it is possible that the crucial variable is the interval between the onset of the disease and the initiation of treatment with neuroleptics (Crow et al, 1986). If this were so, there would be an obvious opening for secondary prevention. The problem is to design an ethical experiment that would distinguish between the two hypotheses.

\section{Bipolar disorder}

The heritability of bipolar disorder is even higher than that of schizophrenia but no 'manicdepression gene' has yet been found. Environmental factors are almost certainly also involved, but even less is known of these than in the case of schizophrenia. Tertiary prevention is therefore the only preventive strategy currently available.

\section{Depressive disorders}

Because they are so common (in the American Epidemiologic Catchment Area Programme the one-month prevalence of major depressive disorder was $1.6 \%$ in men and $2.9 \%$ in women) and generally treatable, depressive disorders potentially offer considerable scope for public health measures.

There is good evidence of a genetic element for severe unipolar depression, but no genes have yet been identified. There is also a consistent body of data implicating social stress in the onset of depressive episodes, with raised rates of threatening life events and chronic difficulties, and somewhat weaker evidence implicating inadequate social support (Paykel \& Cooper, 1992). This ought to create useful possibilities for prevention. Unfortunately, the most important life events are bereavements, separations and the failures damaging to self-esteem that are an almost inevitable part of life. The situation is complicated further by evidence that genetic factors contribute to an individual's liability to experience adverse life events and also to whether or not social supports are available to them. Even so, early intervention after bereavement and possibly other stressful events also may reduce subsequent morbidity (Parkes, 1981).

It is well established that a high proportion of all depressions remain undiagnosed, and therefore untreated. In the UK, general practitioners fail to recognise about $50 \%$ of the psychiatric disorders presenting to them, a high proportion of them depressions, and the reasons why have been studied in detail (Goldberg \& Huxley, 1980). Whether more effective detection and treatment are regarded as secondary (early diagnosis and treatment) or tertiary (minimising disability) prevention, they are clearly worthwhile objectives and were the principal aims of the recent Defeat Depression Campaign mounted by the Royal Colleges of General Practitioners and Psychiatrists (Paykel \& Priest, 1992).

Postnatal disorders provide particularly good opportunities for prevention which are not yet being adequately exploited. Women with a previous history of puerperal psychosis or of bipolar disorder are at very high risk (about 1 in 3) of developing a puerperal psychosis after subsequent childbirth. There is some evidence that lithium started immediately after delivery reduces this risk (Stewart et al, 1991) but no adequate random-allocation trial has yet been carried out. About $10 \%$ of mothers develop clinically significant depressive symptoms in the early months after childbirth; affected women can be detected by a simple questionnaire (the Edinburgh Postnatal Depression Scale; see Holden, 1994) and it has been shown that counselling by a health visitor can alleviate their symptoms (Holden, 1994). Yet despite the fact that postnatal depression may have enduring emotional and cognitive effects on the infant, most of these depressions remain undiagnosed and untreated.

\section{Suicide and parasuicide}

Although there is good evidence from retrospective studies of suicides that at least $90 \%$ of those who take their own lives were suffering from a psychiatric disorder (most commonly depression) shortly before their death, there is remarkably little evidence that more effective treatment of depression lowers suicide rates. Neither the introduction of electroconvulsive therapy nor the 
advent of tricyclic antidepressants was followed by any reduction in suicides, and our national suicide rate is much the same now as it was at the beginning of the century when there were no effective therapies or even psychiatric services apart from institutional care for melancholia. Indeed, in women the suicide rate is considerably higher now than it was then. However, there is now one study showing that more effective treatment of depression can reduce suicides. Provision of a special educational programme on depression for the general practitioners on the Swedish island of Gotland resulted in increased prescribing of antidepressants and a fall, albeit temporary, in the suicide rate on the island (Rutz et al, 1989).

Most of the well-documented influences on national suicide rates are social and cultural, or concern the availability of means of committing suicide. The increased social cohesion associated with war is consistently associated with a reduction in suicides, and both economic recession and dramatic accounts of suicides (real or fictional) in the media are accompanied by an increase. Perhaps more surprisingly, the removal of (highly lethal) carbon monoxide from domestic gas in the 1960s was associated with a $30 \%$ reduction in the British suicide rate, which lasted for nearly two decades (Kreitman, 1976). This evidence that removal of one means of committing suicide does not immediately result in substitution of another underlies several current proposals for reducing British suicide rates. As a high proportion of male suicides are now due to poisoning by car exhaust gases, it has been suggested that the shape of exhaust pipes should be altered to make it harder to fit a rubber tube over them and that catalytic converters (which remove a high proportion of the carbon monoxide) should be fitted to all cars. (The latter is now taking place, but as a European measure to combat atmospheric pollution rather than as anti-suicide initiative.) Because an increasing proportion of female suicides are due to overdoses of paracetamol, the Medicines Control Agency, in a widely circulated letter, has recently proposed restricting the number of paracetamol tablets that can be sold both by ordinary shops and by pharmacies and the provision of stronger warning labels. Others have suggested that paracetamol should be available only on prescription, or as individually wrapped tablets, or in combination with its antidote, methionine.

Many suicides have a past history of parasuicide. Unfortunately no management strategy for parasuicide has yet been shown to reduce the subsequent suicide rate.

\section{Alcohol and drug misuse and dependence}

Two fundamentally different but complementary means of primary prevention are available: reduction of supply and reduction of demand. Determined efforts are made by the international community to reduce the supply of heroin, cocaine and cannabis by destroying crops or providing Third World farmers with financial incentives to grow other crops instead. Customs and excise departments attempt to intercept smuggled drugs in transit and police forces arrest those found in possession. Controls on prescribed drugs are also important. At various times in the past 20 years the medical profession has taken initiatives to restrict prescribing of barbiturates and amphetamines and, more recently, of temazepam and other benzodiazepines and of buprenorphine (Temgesic). There are also long-established, but steadily weakening, controls on the supply of alcoholic beverages. They cannot be legally sold to children under the age of 18 and their sale is restricted to particular licensed premises (on and off licenses) and times of the day.

A variety of means of reducing demand are also available. The fact that consumption of heroin, cocaine, ecstasy and cannabis is illegal and subject to draconian penalties presumably reduces demand, although it does not prevent $20 \%$ of British university students smoking cannabis every week and $33 \%$ experimenting with other illicit drugs as well (Webb et al, 1996). The excise and other duties levied on alcoholic beverages also reduce demand and it is well established that even quite small changes in the price of alcoholic beverages relative to incomes result in changes in per capita consumption, which in turn generate corresponding changes in indices of alcohol-related harm such as cirrhosis mortality rates, convictions for public drunkenness and drunken driving, and hospital admissions for alcohol dependence (Special Committee of the Royal College of Psychiatrists, 1986). Unfortunately, at present any increase in the excise duty on alcoholic beverages in the UK is likely to stimulate further imports, legal and illegal, from northern France. Education campaigns are also widely employed in the hope of reducing demand for illegal drugs, including heroin and ecstasy, and to curb irresponsible drinking, but there is little evidence that they are effective.

History teaches us that attempts to control the use of illegal drugs simply by controlling their 
supply are doomed to fail. However determined governments may be, their efforts will be thwarted by an increasingly sophisticated international criminal network attracted by the high profits to be made from drug smuggling. It is equally apparent that demand cannot be reduced much by educational campaigns. In Britain and elsewhere, drug abuse flourishes in deprived neighbourhoods with poor schools, high unemployment and high levels of marital breakdown and juvenile and adult delinquency, and it does not wane until deprivation and unemployment have been alleviated. History also teaches us that widespread alcohol misuse can be successfully curbed by legislation if, but only if, the government of the day is resolute and has the backing of the majority of its citizens. The British government has acted decisively and effectively twice in the past 250 years: first in the 18th century to curb the havoc being wrought in urban slums by the distilled spirit, gin (the 1751 Act 'for more effectually restraining the retailing of distilled spirituous liqueurs' and the Disorderly Houses Act of 1752), and then during the First World War to control the damaging effects of widespread drunkenness on the war effort (Lloyd George's Defence of the Realm Act of 1915). On the other hand, the attempts of the American and other governments to impose prohibition in the 1920s, and Mr Gorbachov's attempt to reduce consumption of vodka in Russia in the 1980s, ended up as costly failures, despite impressive initial successes, because too high a proportion of the population resented the new controls and connived in the growth of a black market.

Against this background it is important that effective secondary prevention of alcohol dependence is possible. Several well-designed randomised trials, based on patient populations in either a general hospital or a general practice setting, have demonstrated that people who drink excessively can be identified with simple self-report questionnaires such as the 10 -item AUDIT, and that brief interventions, usually by a specially trained nurse, can be effective in enabling these heavy drinkers to reduce their consumption before they develop serious dependence (Freemantle et al, 1993).

\section{Post-traumatic stress disorder and phobic disorders}

It is only in the past 30 years that the scale and chronicity of psychiatric symptoms after exposure to disasters and other overwhelmingly traumatic events have become apparent. This awareness has been followed by attempts to prevent or mitigate these adverse sequelae, usually by some form of counselling or debriefing immediately after the traumatic incident (e.g. Hoiberg \& McCaughey, 1984). There have been few adequate evaluations of these therapies, for it is singularly difficult, for both practical and emotional reasons, to organise a random allocation trial in the immediate aftermath of a horrifying disaster. There is now, however, some emerging evidence that encouraging disaster survivors to recount and relive their traumatic experience, and 'debriefing' the victims of road traffic accidents, may actually exacerbate rather than reduce long-term sequelae, emphasising the urgent need for more rigorous evaluations (e.g. Hobbs et al, 1996).

More common traumatic events, like car and aeroplane crashes and climbing accidents, and even 'near misses', often lead to mounting anxiety and phobic avoidance on return to the activity or situation concerned. It is now well established that rapid resumption of the activity (e.g. driving or flying) is the best way of preventing the development of an incapacitating phobia, and air forces throughout the world act accordingly.

\section{Eating disorders}

The prevalences of anorexia nervosa and bulimia nervosa have increased dramatically in most Western countries since the 1960s. Although the aetiology of both disorders is complex, there is little doubt that the prevailing concept of female beauty, which requires influential role models to be slender to the point of emaciation, has played a large part in this huge increase in prevalence, and both conditions are still rare in cultures in which buxom women are still admired and envied.

Although psychiatrists can hardly hope to influence the Western world's concept of beauty, they may still exert a beneficial preventive role by counselling the staff and pupils of ballet and modelling schools and similar institutions where the pressures to be slim are particularly likely to lead to pathological eating habits.

\section{Childhood disorders}

Childhood and adolescence have raised the highest hopes of educational and psychotherapeutic interventions and also witnessed the greatest 
disappointments. None the less, some childhood interventions have $u$ doubtedly been successful in reducing even long-term morbidity. Although the optimism of the Mental Hygiene Movement of the 1920s and 1930s proved unfounded, longterm follow-up of children from the American Headstart programmes of the 1960s have shown that, although the improvements in IQ were relatively short-lived, there were enduring gains in social functioning and emotional maturity, in delinquency rates and in employment records (Darlington et al, 1980). Similarly, although schoolbased programmes in the USA to reduce suicide and parasuicide rates in teenagers by heightening awareness of the problem, enhancing coping skills and providing crisis intervention services have had little success (Tayler et al, 1992), there is evidence from this country that well-run schools can have beneficial effects on the adult adjustment and functioning of girls brought up in institutions (Quinton et al, 1984).

A few other programmes have also demonstrated benefits from attempts at secondary prevention. Kolvin et al (1981), for example, identified maladjusted children by screening school populations, and then compared four different interventions (combined parent counselling and teacher consultation; group therapy or play groups; nurturing in young children; and behaviour modification for older children) with no intervention. Significant benefits were found for all four interventions, particularly for nurturing and behaviour modification.

\section{General well-being}

Surveys of the prevalence of common psychiatric symptoms in the general population using wellvalidated instruments like the General Health Questionnaire (Goldberg, 1972) consistently show that symptom levels are higher in women than in men, higher in urban than in rural areas, higher in the unemployed than in people with jobs, higher in people in stressful occupations like medicine and nursing than other less demanding jobs, and higher in people who are inactive than in those who take regular exercise. They are also higher in single than in married men, but higher in married than in single women. Of all these relationships the one which offers the most obvious public health opportunity is regular exercise. The difference between the questionnaire scores of those who take regular exercise and those who take almost none is clinically as well as statistically significant both in adolescents and in adults (Steptoe \& Butler, 1996) and has also been demonstrated in randomised controlled trials that allocated inactive people to training or control conditions (Steptoe, 1992). The fact that regular exercise also protects against coronary artery disease, non-insulin dependent diabetes, obesity and osteoporosis is, of course, an added bonus.

\section{Conclusions}

On the basis of present knowledge there are many ways in which the incidence or prevalence of a wide range of different psychiatric disorders could be lowered. However, none of these interventions is obviously capable of reducing incidence to the point at which therapeutic services could be withdrawn, or even markedly reduced. Moreover, for most of these interventions the levers of power are not in the hands of psychiatrists. They are in the hands of legislators, safety engineers, obstetricians, paediatricians, geneticists, general practitioners and the general public. There are, however, a number of areas in which psychosocial interventions of various kinds have been shown to be effective. The prevention of alcohol dependence, of postnatal psychiatric disorders, of bereavement reactions and of PTSD, and ameliorating the long-term consequences of childhood deprivation, have all been shown to be feasible. This evidence should lead to action far more widely (Box 3). This applies particularly to the relatively simple, inexpensive measures available for detecting and combating hazardous drinking habits and postnatal depression. However, one of

Box 3. Proven opportunities for primary or secondary prevention which are not yet properly exploited by psychiatrists

Detection of hazardous drinking by simple questionnaires followed by advice

Detection of postnatal depressions by a questionnaire followed by counselling

Prevention of puerperal psychoses in highrisk pregnant women

Prevention of pathological bereavement reactions, and possibly of post-traumatic stress disorders, by early interventions

Ameliorating the long-term consequences of childhood deprivation 
Box 4. Summary

Primary or secondary prevention is possible for many psychiatric disorders, and some (pellagra and general paralysis of the insane) have already been eliminated. For most effective interventions, however, the levers of power are in the hands of nonpsychiatrists-legislators, safety engineers, obstetricians, paediatricians, geneticists, general practitioners and the general public. Few psychiatric interventions have been shown to be effective, except in tertiary prevention, but this is probably because too few random allocation trials have been performed.

the most important contributions psychiatrists can make to public health at present - apart from their long-established role in minimising relapse rates in schizophrenia and unipolar and bipolar affective disorders - is to help design and carry through the painstaking random allocation trials that are needed to demonstrate which psychological and social interventions are effective in which circumstances, and how cost-effective they are (Box 4).

\section{Further reading}

CRAG Working Group on Mental Illness (1995) Primary Prevention of Mental Health Problems. Edinburgh: The Scottish Office.

Royal College of Psychiatrists (1993) Prevention in Psychiatry. Council Report CR21. London: Royal College of Psychiatrists.

\section{References}

Crow, T. J., Macmillan, J. F., Johnson, A. L., et al (1986) The Northwick Park study of first episodes of schizophrenia. II. British Journal of Psychiatry, 148, 120-127.

Darlington, R. B., Royce, J. M., Snipper, A. S., et al (1980) Preschool programs and later school competence of children from low income families. Science, 208, 202-208.

Freemantle, N., Gill, P., Godfrey, C., et al (1993) Brief interventions and alcohol use. Quality in Health Care, 2, 267-273.

Geddes, J. R. \& Lawrie, S. M. (1995) Obstetric complications and schizophrenia: a meta-analysis. British Journal of Psychiatry, 167, 786-793.

Goldberg, D. P. (1972) The Detection of Psychiatric Illness by Questionnaire (GHQ). Maudley Monograph 21. London: Oxford University Press.

- \& Huxley, P. (1980) Mental Illness in the Community, pp. 73156. Oxford: Oxford Medical Publications.
Hobbs, M., Mayou, R., Harrison B., et al (1996) A randomised controlled trial of psychological debriefing for victims of road traffic accidents. British Medical Journal, 313, 1438-1439.

Hoiberg, A. \& McCaughey, B. G. (1984) The traumatic after effects of collision at sea. American Journal of Psychiatry, 141, 70-73.

Holden, J. (1994) Can non-psychotic depression be prevented? Use and misuse of the Edinburgh Postnatal Depression Scale. In Perinatal Psychiatry (eds J. Cox \& J. Holden), pp. 54-81. London: Gaskell.

Kolvin, I., Garside, R. E. Nichol, A. R., et al (1981) Help Starts Here: The Maladjusted Child in the Ordinary School. London: Tavistock.

Kreitman, N. (1976) The coal gas story: UK suicide rates 19601971. British Journal of Preventive and Social Medicine, 30, 86-93.

Myhrman, A., Rantakallio, P., Isohanni, M., et al (1996) Unwantedness of a pregnancy and schizophrenia in the child. British Journal of Psychiatry, 169, 637-640.

Parkes, C. M. (1981) Evaluation of a bereavement service. Journal of Preventive Psychiatry, 1, 179-188.

Paykel, E. S. \& Cooper, Z. (1992) Life events and social support. In Handbook of Affective Disorders (2nd edn) (ed. E. S. Paykel), pp. 149-170. Edinburgh: Churchill Livingstone.

- \& Priest, R. G. (1992) Recognition and management of depression in general practice: consensus statement. British Medical Journal, 305, 1198-1202.

Quinton, D., Rutter, M. \& Liddle, C. (1984) Institutional rearing, parenting difficulties and marital support. Psychological Medicine, 14, 107-124.

Rutz, W., van Knorring, L. \& Walinder, J. (1989) Frequency of suicide on Gotland after systematic postgraduate education of general practitioners. Acta Psychiatrica Scandinavica, 80, 151-154.

Special Committee of the Royal College of Psychiatrists (1986) Alcohol: Our Favourite Drug, pp. 104-125. London: Tavistock

Steptoe, A. (1992) Physical activity and psychological wellbeing. In Physical Activity and Mental Health (ed. N. G. Norgan), pp. 207-229. Cambridge: Cambridge University Press.

- \& Butler, N. (1996) Sports participation and emotional wellbeing in adolescents. Lancet, 347, 1789-1792.

Stewart, D. E., Klompenhouwer, J. L., Kendell, R. E., et al (1991) Prophylactic lithium in puerperal psychosis: the experience of three centres. British Journal of Psychiatry, 158, 393-397.

Tayler, P. J., Burton, K. \& Kolvin I. (1992) Suicidal behaviour in children and adolescents. In Recent Advances in Clinical Psychiatry, no. 7 (ed. K. Granville-Grossman). Edinburgh: Churchill Livingstone.

Wald, N. J., Kennard, A., Densem, J. W., et al (1992) Antenatal maternal serum screening for Down's syndrome: results of a demonstration project. British Medical Journal, 305, 391-394.

Webb, E., Ashton, C. H., Kelly, P., et al (1996) Alcohol and drug use in UK university students. Lancet, 348, 922-925.

\section{Multiple choice questions}

1. The following are examples of secondary prevention:

a keeping people with schizophrenia on longterm neuroleptics

b treating manic illnesses with lithium instead of neuroleptics

c screening for cancer of the breast

d screening in general practice for hypertension

e amniocentesis and termination of pregnancy for Down's syndrome. 
2. The following have already been demonstrated to be effective me. is of primary or secondary prevention in psychiatry:

a reducing the aluminium content of drinking water

b training health visitors to detect and treat postnatal depressions

c promoting positive mental health in the general population

d screening people with a family history of bipolar disorder for the gene on chromosome II

e warning schoolgirls of the dangers of paracetamol.

3. The following are frequently associated with a rise in the suicide rate:
a lurid media descriptions of suicides
b civil wars
c rising unemployment
$\mathrm{d}$ onset of winter
e rising national divorce rates.

4. The following measures have been shown to reduce the ill effects associated with alcohol consumption:

a educational campaigns warning people not to drink more than the 'sensible limits'

$b$ reducing male unemployment

c the 1915 Defence of the Realm Act

d increasing the excise duty on alcoholic beverages

e the 1968 Road Traffic Act.
5. Which of the following statements about childhood psychiatric morbidity is true:

a the American Headstart programmes failed to produce lasting benefits

b there is still no evidence that psychiatric interventions with maladjusted children are effective

c school refusal is best treated with cognitive therapy

d if we had more child psychotherapists, most adult neurotic illness could be prevented

e screening for fragile $X$ could greatly reduce the number of girls born with learning disabilities.

\begin{tabular}{|c|c|c|c|c|}
\hline \multicolumn{5}{|c|}{ MCQ answers } \\
\hline 1 & 2 & 3 & 4 & 5 \\
\hline a F & a $F$ & a $\mathrm{T}$ & a F & a $F$ \\
\hline b F & b $\mathrm{T}$ & b F & b F & b F \\
\hline c $\mathrm{T}$ & c $\mathbf{F}$ & c $\mathrm{T}$ & c $\mathrm{T}$ & c $\mathrm{F}$ \\
\hline d $\mathrm{T}$ & d F & d F & d $T$ & d F \\
\hline e $F$ & e F & e $F$ & e $T$ & e $\mathrm{F}$ \\
\hline
\end{tabular}

